\title{
FORMAÇÃO TÉCNICA DE AGENTE COMUNITÁRIO INDÍGENA DE SAÚDE: UMA EXPERIÊNCIA EM CONSTRUÇÃO NO RIO NEGRO
}

\author{
TECHNICAL TRAINING OF INDIGENOUS COMMUNITY HEALTH AGENTS: AN UNDER \\ CONSTRUCTION EXPERIENCE IN RIO NEGRO
}

\author{
Luiza Garnelo ${ }^{1}$ \\ Esron Rocha ${ }^{2}$ \\ Paulo Peiter ${ }^{3}$ \\ Sully Sampaio ${ }^{4}$ \\ Elciclei Santos ${ }^{5}$ \\ Ana Lúcia Pontes ${ }^{6}$ \\ Anakeila Stauffer ${ }^{7}$
}

Resumo Este relato descreve a experiência de formação técnica profissionalizante de 250 agentes comunitários indígenas de saúde vinculados ao Distrito Sanitário Especial Indígena do Rio Negro, no estado do Amazonas. A iniciativa promove a elevação da escolaridade e o respeito às especificidades culturais dos alunos. Os eixos pedagógicos 'cultura', 'território', 'política', 'cuidado', 'informação', 'educação' e 'planejamento em saúde' estruturam uma matriz curricular operacionalizada por meio do ensino pela pesquisa, multilinguismo, multidisciplinaridade e intersetorialidade, em consonância com os princípios da educação escolar indígena, da atenção diferenciada à saúde dos povos indígenas e dos referenciais curriculares de formação técnica de agente comunitário de saúde. O processo pedagógico estimula a obtenção de habilidades e competências para diagnosticar e monitorar a situação de saúde e condições de risco e vulnerabilidade das populações indígenas rionegrinas, para subsidiar ações de prevenção, promoção, tratamento e reabilitação nas diversas fases da vida e desenvolver ação política e comunitária nas lutas pela melhoria da saúde. Resultados preliminares da experiência mostram o reconhecimento e fortalecimento do trabalho dos agentes indígenas de saúde, a melhoria da qualidade do trabalho em saúde na comunidade e a maior satisfação dos líderes do movimento indígena pela ampliação do acesso à educação qualificada e diferenciada.

Palavras-chave saúde indígena; agentes comunitários de saúde; formação profissional em saúde.
Abstract This report describes the experience of the technical professional training of 250 indigenous community health agents linked to the Special Indigenous Sanitary District of Rio Negro in the state of Amazonas. The initiative promotes the increase of the level of education and the respect towards the specific cultural features of the students. The main pedagogical elements, 'culture', 'territory', 'policy', 'care', 'information', 'education', and 'health planning' form a curriculum matrix that is operationalized through teaching based on research, multilinguism, multidisciplinarism, and intersectoralism, in tune with the principles of indigenous education, the special attention to the health of indigenous peoples, and of curriculum references of the technical training of community health agents. The pedagogy process fosters the acquisition of skills and competencies to diagnose and monitor health conditions and the risk and vulnerability of indigenous populations, in order to support preventive actions, promotion, treatment, and rehabilitation at the different stages of life, and develop political and communal action in the fight for better health. Preliminary results of the experience show the recognition and strengthening of the indigenous health agents work, the improving the quality of health work in the community, and the highest level of satisfaction of the leaders of the indigenous movement, by broadening the access to a qualified and specialized education.

Keywords indigenous health; community health agents; professional training in health. 


\section{Introdução}

A região do Alto Rio Negro tem uma população de 38 mil indígenas em cerca de 600 aldeamentos distribuídos em 12 milhões de hectares de terras demarcadas, em três municípios do estado do Amazonas: São Gabriel da Cachoeira, Santa Isabel e Barcelos (Brasil, 2003). A localização das aldeias segue o curso do rio Negro e afluentes, expressando formas de territorialização e de diferenças culturais entre os povos que ali vivem, ainda que estes tenham uma história ancestral de interações mútuas que moldaram um sistema cultural próprio, contrastivo com as povoações não-indígenas implantadas no processo de colonização da região.

A população indígena no Alto Rio Negro enfrenta uma situação de grande vulnerabilidade social e sanitária, expressa pela dificuldade de acesso aos recursos alimentares, pelas elevadas taxas de natalidade e de mortalidade infantil e por um perfil epidemiológico marcado pela excessiva frequência de doenças infectocontagiosas e, em particular, as de veiculação hídrica, que coexistem com a precária interiorização das políticas públicas nas aldeias. Esse contexto socioeconômico e demográfico se reproduz num cenário natural adverso, marcado pelas longas distâncias e por acidentes geográficos, como as grandes cachoeiras que dificultam o deslocamento fluvial (principal forma de transporte nessa região) e prejudicam o acesso ao atendimento em saúde e educação, assim como outras iniciativas voltadas para a melhoria das condições de vida.

A literatura antropológica aponta um contexto multiétnico e multilinguístico com 19 grupos étnicos distribuídos nesse amplo espaço geográfico. Dentre as características comuns a essas sociedades, são dignas de nota: a grande dispersão espacial dos assentamentos; a baixa densidade demográfica por $\mathrm{km} 2$ (estratégias desejáveis num ecossistema carente de nutrientes); e a grande capacidade de gestão e manutenção de iniciativas comunitárias de desenvolvimento sustentável e de interagir com instituições públicas e privadas através de um aprimorado associativismo indígena. Esta última característica tem possibilitado a captação de recursos e o desenvolvimento de iniciativas que favorecem o acesso a bens e serviços da sociedade nacional brasileira (Wright, 1992; Garnelo, 2003).

A baixa escolaridade tem dificultado o aprimoramento da capacidade de gestão e de organização política desses povos. Ainda que a população rionegrina tenha um grau de escolaridade mais elevado do que a média entre os povos indígenas do país, seu maior contingente de escolarizados concluiu apenas o Ensino Fundamental. Por exemplo, do total de agentes indígenas de saúde (AIS) que atuam no Alto Rio Negro, apenas 3,6\% concluíram o Ensino Médio, estando os outros 96\% impossibilitados de acessar cursos de ensino técnico profissionalizante, pois a legislação brasileira 
exige do profissional técnico de nível médio em saúde a certificação concomitante, ou prévia, de Ensino Médio.

Em estudo sobre a capacitação dos agentes de saúde, Rocha (2007) verificou que no período de 1990 a 2006 eles receberam 12 treinamentos curtos, ofertados por diversas instituições, totalizando uma carga horária de 912 horas. Nenhum destes cursos dispunha de conteúdos programáticos voltados para a especificidade das culturas locais. Limitavam-se a temas biomédicos descoordenados entre si, incapazes de propiciar a elevação da escolaridade e a profissionalização. Tais antecedentes mobilizaram as lideranças indígenas na demanda de formação profissional para os AIS do Alto Rio Negro.

\section{O processo de construção do curso}

As associações indígenas do Alto Rio Negro desenvolveram vários projetos no campo da educação indígena. Uma das grandes conquistas desse movimento foi a formação profissional dos professores indígenas. Entretanto, tais avanços não se repetiram no campo da saúde, apesar da implantação, em 1999, do subsistema de saúde indígena, localmente representado pelo Distrito Sanitário Especial Indígena (Dsei) do Rio Negro (Rocha, 2007).

Em 2007, as lideranças indígenas empreenderam diversas iniciativas que visavam a garantir a formação dos AIS. Inseriram tal demanda no plano trienal do Dsei para 2008-2010; em seguida, obtiveram a resolução n. ${ }^{\circ}$ 001/ 2007 do Conselho Distrital de Saúde do Rio Negro, que recomendou a formação profissional dos AIS no prazo máximo de cinco anos. A implementação da proposta foi buscada através de parcerias institucionais capazes de desenvolver o processo formador. O Instituto Leônidas e Maria Deane (ILMD), da Fiocruz-AM, foi a parceira escolhida para viabilizar a formação. Este buscou apoio da Escola Politécnica de Saúde Joaquim Venâncio (EPSJV), que detém expertise em ensino e pesquisa no campo da educação profissional em saúde, com destaque para o Ensino Médio integrado à educação profissional.

No segundo semestre de 2007, pesquisadores de ambas as instituições começaram a desenvolver a proposta de formação dos AIS. Para este fim, articularam uma rede de parcerias que congregou a Federação das Organizações Indígenas do Alto Rio Negro, o Dsei do Alto Rio Negro, a prefeitura, as secretarias municipais de Educação e de Saúde de São Gabriel da Cachoeira, a Secretaria Estadual de Educação do Amazonas e o Departamento de Saúde Indígena (Desai/MS). Tal rede visava a garantir o processo formador, assim como a certificação dos alunos ao final do curso.

O caráter participativo da iniciativa foi garantido através de reuniões, oficinas de trabalho e seminários. Tais eventos envolveram os parceiros 
institucionais, agentes de saúde, professores e outras lideranças indígenas, além de comunitários em geral. A partir deles, estabeleceram-se os passos necessários à implantação de um programa de Ensino Médio integrado, dirigido à totalidade dos AIS que atuam no Dsei Rio Negro. Também estimularam a troca de experiências entre os participantes e promoveram a reflexão sobre os avanços e limites das capacitações feitas em anos anteriores, bem como sua potencial aplicação no processo formador a ser implementado.

Em que pese a existência de um subsistema de saúde indígena no Sistema Único de Saúde (SUS) voltado para prover atenção diferenciada para as etnias que vivem no Brasil, não existem marcos legais para uma formação profissional específica para essas minorias. Assim sendo, a equipe buscou respaldo na legislação e nos parâmetros curriculares que orientam a formação de agentes comunitários não-indígenas no SUS.

Os serviços de saúde do SUS e do seu subsistema de saúde indígena, implementado localmente através dos Dsei, partilham a atenção primária à saúde como fundamento comum. Assim sendo, a equipe de trabalho considerou factível o uso dos parâmetros orientadores da formação de agente comunitário de saúde (ACS), para operacionalizar o processo formativo no Alto Rio Negro. Para tal fim, congregou as orientações fornecidas pelo Referencial Curricular para Curso Técnico de Agente Comunitário de Saúde, proposto pelos ministérios da Saúde e da Educação (Brasil, 2005), pelo documento "Educação profissional básica para agentes indígenas de saúde", elaborado pela Fundação Nacional de Saúde (Funasa) (Brasil, 1999), e pelo Curso Técnico de Vigilância em Saúde, desenvolvido pela EPSJV (Gondim, 2009; Monken, 2008; EPSJV, 2005).

Com base nesses marcos, o grupo elaborou a proposta de Curso Técnico de Agentes Comunitários Indígenas de Saúde, que se apoia, simultaneamente, nos princípios da educação indígena e da atenção diferenciada à saúde dos povos indígenas e nos citados referenciais curriculares de formação técnica de ACS. Para demarcar o novo momento, propôs-se a adoção do termo agente comunitário indígena de saúde (Acis), em substituição à designação anterior, de agente indígena de saúde (AIS).

Uma versão inicial da proposta foi apresentada em assembleia dos AIS do Alto Rio Negro, em março de 2008, que também contou com a participação das diversas parcerias envolvidas no projeto. Após as discussões e sugestões de aprimoramento, uma nova versão foi sistematizada e submetida ao Conselho Estadual de Educação do Amazonas.

Paralelamente, prosseguiram as negociações com as secretarias municipal e estadual de Educação, visando a garantir os cursos de Ensino Fundamental e Médio que possibilitassem elevar a escolaridade dos agentes. Tais iniciativas foram bem-sucedidas e estão sendo implementadas. 


\section{Princípios orientadores da proposta}

Os princípios orientadores do curso priorizam o ensino baseado em pesquisas, desenvolvido nas próprias aldeias indígenas, com valorização e revitalização de saberes e práticas tradicionais indígenas, e comprometido com a diferenciação cultural e com o protagonismo político dos AIS, com o ensino integrado que agregue formação técnica à elevação da escolaridade. O curso também foi entendido como capaz de gerar um modelo de formação para AIS, a ser utilizado - com as devidas adaptações às diversas realidades culturais - em outras regiões do Amazonas ou do país.

As premissas da educação escolar indígena, ou seja, especificidade, interculturalidade, bilinguismo, multidisciplinaridade, dialogicidade, pedagogia crítica, ensino pela pesquisa e respeito às singularidades culturais indígenas e à história do processo de colonização também orientam o perfil do curso (Castro, 2005).

No campo da saúde pública, o marco orientador é representado pela promoção e pela vigilância em saúde, entendidas como redefinições das práticas sanitárias vigentes no sistema de saúde. Elas são concebidas como modelos de atenção conformados por combinações tecnológicas que visam ao controle de riscos e danos à saúde. Têm por objeto as relações entre os modos de vida de grupos sociais e as formas diversificadas de expressão do processo saúde-doença.

Nos cenários sociais, a promoção da saúde surge como alternativa de empoderamento dos sujeitos para intervenção nos determinantes e condicionantes do processo saúde-doença. Nessas circunstâncias, os processos educativos operam como via privilegiada para operacionalizar ações qualificadas e efetivas de promoção e manutenção da saúde. Na dimensão políticogerencial, a vigilância em saúde valoriza processos de trabalho capazes de intervir sobre os problemas de saúde em diferentes momentos de sua determinação, e não apenas sobre os já instalados, como processos patológicos, tal como ocorre nas práticas de medicina curativa (Teixeira, Paim e Vilasboas, 1998; Teixeira e Solla, 2006).

As propostas de vigilância em saúde fertilizam as concepções pedagógicas presentes no conceito de 'educação para o trabalho em saúde', estimulando processos formativo-educativos que valorizam o protagonismo político, a intervenção intersetorial e o compromisso com a redução das desigualdades sociais. Elas instituem, também, o monitoramento da situação de saúde de populações vinculadas a determinados territórios (Ceccim e Ferla, 2008-2009). No caso da população do Alto Rio Negro, os espaços das ações de vigilância em saúde a serem desenvolvidas pelos agentes de saúde equivalem aos territórios tradicionais de seus clãs de origem.

Diversos estudos antropológicos sobre as culturas rionegrinas tratam das redes de sentidos sobre a origem e classificação dos processos de doença 
e os sistemas tradicionais de cura e cuidados (Garnelo, 2003, 2007; Garnelo e Wright, 2001; Buchillet, 1995). Igualmente relevantes são as investigações sobre a organização social e política desses povos (Wright, 1992; Hill, 1984). São informações que propiciam o entendimento das formas de ocupação territorial, de acesso às fontes alimentares e a outros meios de sobrevivência, do exercício do poder político e das iniciativas comunitárias de autogestão e desenvolvimento sustentável que subsidiam o protagonismo político indígena e oferecem suporte ao desenvolvimento de ações de vigilância em saúde.

Os pressupostos do processo pedagógico aqui relatado valorizam a inserção dos educandos no seu campo de trabalho, tomando-a como uma modalidade de educação profissional que se estrutura a partir do eixo laborativo. A educação profissional deve ser vista não como mera reprodução de técnicas e procedimentos, mas como busca de entendimento dos fundamentos do conhecimento científico e das técnicas e dos procedimentos dele derivados (Ciavatta, 2005). Ela propicia a aquisição e o desenvolvimento de conhecimentos teóricos, técnicos e operacionais, mas sem descurar da capacidade do educando atuar como sujeito autoconstrutor e transformador das condições atualmente existentes na vida social. Esta relação entre educação e transformação deve ser também fruto de discussão, evitando-se a compreensão do processo formativo como um 'processo curativo' sobre os indivíduos destinatários da formação, transformando o trabalho de formação numa 'ciência da certeza' (Canário, 2003).

Os processos de aprendizado compreendem o educando-trabalhador indígena como sujeito de conhecimentos, produzido na/pela cultura. Ele atua numa realidade onde estão presentes os aspectos econômicos, políticos, históricos, culturais, científicos, técnico-operacionais e ambientais, ou seja, sua ação se dá em uma totalidade. O processo de ensino-aprendizagem considera, portanto, que os conteúdos devem ser contextualizados, propiciando refletir sobre a realidade de forma crítica, para além do senso comum.

\section{O curso técnico de agente comunitário indígena de saúde}

Na organização do projeto pedagógico, uma das opções possíveis teria sido a formação de técnicos de enfermagem, seguindo o modelo de experiências já desenvolvidas em outras áreas indígenas no país. Porém, o entendimento de ser esta uma formação marcada pelo modelo de assistência hospitalar levou a equipe a buscar uma alternativa mais congruente com as necessidades e o perfil sociossanitário das comunidades beneficiárias do processo formador. Assim, a proposta da formação de agentes comunitários surgiu como alternativa adequada para a prestação de serviços de saúde indígena no Alto Rio Negro, desde que houvesse um esforço coletivo de adaptar os 
parâmetros curriculares vigentes à diversidade cultural existente no cenário sociopolítico em que o processo formador se desenvolve.

A equipe de gestão do curso optou pelo estabelecimento de relações de ensino-aprendizado pautadas pela valorização da cultura das sociedades de origem dos educandos e pela articulação de conhecimentos do campo da saúde pública com os saberes, práticas e modos de vida próprios às culturas indígenas. $\mathrm{O}$ aprendizado por competências, tomado numa perspectiva contra-hegemônica, partiu do processo de trabalho, associou-o à educação básica e relacionou conhecimentos científicos, técnicos, operacionais, organizacionais com o mundo do trabalho dos Acis e com sua cultura, instituindo um processo formador de caráter totalizante (Ramos, 2003).

O desafio de ofertar não apenas um curso de formação profissional, mas também de construir um processo de trocas interculturais, instituiu um diálogo permanente entre membros de culturas estranhas entre si (educadores e educandos), tornadas progressivamente mais familiares à medida que a relação ensino-aprendizado se aprofunda.

A oferta de Ensino Médio integrado à formação técnica profissionalizante demandou uma carga horária total de 3.240 horas de curso, divididas em 1.800 horas de escolaridade regular, sendo o restante voltado para a formação profissional; na distribuição dessas últimas, prevê-se 240 horas para a prática profissionalizante. O processo formador está distribuído em cinco pólos de formação (Alto Rio Negro/Xié/Baixo Içana, Médio/Alto Içana e Aiari, Alto Waupés/Papuri, Baixo Waupés/Tiquié, Médio Baixo Rio Negro e Estrada SGC-Cucuí), como expresso na Figura 1.

Figura 1

Distribuição de pólos de formação dos agentes comunitários indígenas de saúde

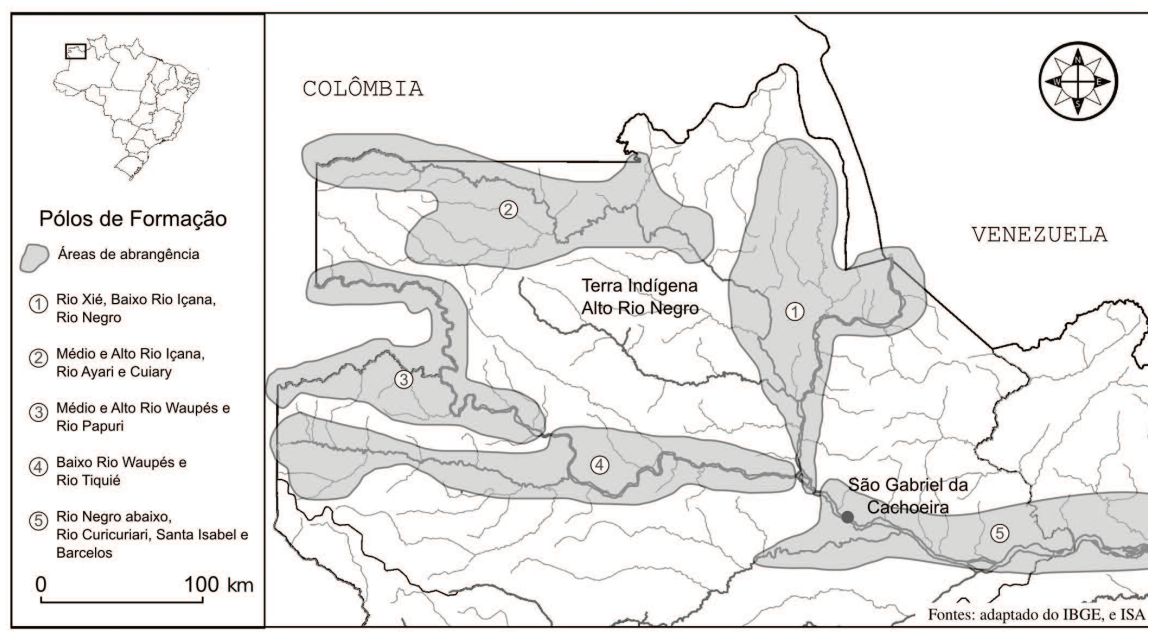

Fonte: Instituto Socioambiental, MMA/SBF, 2001 
A divisão dos pólos de formação expressa o respeito às estratégias de territorialização dos grupos étnicos partícipes do processo educativo. A interiorização dos pólos também visa a facilitar o acesso dos educandos ao curso e a tornar a frequência às aulas e a outras atividades extraclasse algo factível para trabalhadores da saúde compromissados com postos comunitários de trabalho e com a subsistência de suas próprias famílias. Além disso, os agentes de saúde representam a única alternativa de atendimento à saúde em diversas comunidades, o que dificulta o afastamento dos seus locais de trabalho, por longos períodos, para acompanhar os cursos.

A matriz curricular do Ensino Médio se organiza através das diversas áreas do conhecimento, tais como: Linguagens, Códigos e suas Tecnologias; Ciências da Natureza, Matemática e suas Tecnologias; Ciências Humanas e suas Tecnologias. Deve-se salientar que o curso pretende trabalhar os conteúdos curriculares a partir de temas ou problemas oriundos da realidade local, levantados pelos próprios educandos.

A formação técnica em saúde está estruturada segundo os seguintes eixos curriculares: 'cultura', 'território', 'política', 'cuidado', 'informação', 'educação e planejamento em saúde'. Operacionalmente, ela é dividida em três etapas formativas, nas quais os educandos serão progressivamente inseridos de acordo com a elevação de sua escolaridade, conforme orientação do referencial curricular nacional do curso de ACS (Brasil, 2005).

A primeira etapa de formação inicia pelo conhecimento do território e das sociedades indígenas locais, como subsídio à intervenção sanitária. A segunda etapa é voltada para analisar e identificar situações de risco e vulnerabilidade, propiciando atuação informada e tecnicamente qualificada no território. A terceira e última etapa funda-se na elaboração de propostas de ação comunitária, definidas a partir do planejamento participativo de estratégias de enfrentamento dos problemas e necessidades de saúde, identificadas nas etapas anteriores do processo formador.

Cada etapa formativa tem seus próprios objetivos que podem ser sintetizados em: a) buscar o entendimento das características do ambiente, do território e da vida em sociedade; b) conhecer os modos nativos de vida e as transformações históricas decorrentes do processo colonizatório; c) valorizar os sistemas tradicionais de conhecimento que orientam e informam a organização dos sistemas nativos de cura e cuidados; d) sistematizar informações que propiciem a prestação de cuidados de saúde culturalmente sensíveis e que contribuam para apoiar o protagonismo político dos indígenas como agentes de transformação de suas condições de vida.

Cada etapa formativa contém conteúdos específicos que enfocam, por um lado, os saberes indígenas e, por outro, o conhecimento científico, numa busca de articular os múltiplos pontos de vista contidos na explicação do processo saúde e doença e na ação de saúde. Visam também a propor 
modelos de vigilância em saúde culturalmente adaptados à realidade indígena. Cada um dos eixos estruturantes do processo formativo dispõe igualmente de objetivos pedagógicos específicos, tais como: conhecer estratégias de territorialização e as relações de poder no âmbito do parentesco indígena; entender como essas microterritorializações viabilizam o acesso a recursos ambientais que propiciam a subsistência, imprimem os marcos identitários acionados no contato interétnico e influenciam na delimitação das áreas de abrangência dos serviços de saúde do Dsei Rio Negro.

No âmbito dos sistemas tradicionais de cura e cuidados, há interesse no conhecimento dos processos (materiais e simbólicos) que viabilizam a reprodução da vida social; no entendimento de como se exerce o poder político do parentesco, que delineia as relações intercomunais e com a natureza; em conhecer a produção mítica que oferece substrato ao cogito indígena e guarda importante interação sociossimbólica com a produção da doença e da vida social. O conceito de território, aqui entendido como espaço socialmente produzido e produtor das relações sociais estabelecidas por aquelas sociedades (Santos, 1997), opera como um tema transversal, abordado nos diversos eixos pedagógicos que sustentam o curso.

Ao longo e ao final do processo formativo, espera-se que os educandos sejam capazes de (re)conhecer as condições de vida da população que atendem, bem como as situações que protegem e ameaçam a vida; conhecer recursos de importância alimentar e sanitária em seu território de atuação; coletar, sistematizar e analisar dados e informações do território, visando a sua utilização no monitoramento dos problemas de saúde; compreender o processo de saúde-doença e cuidado, tanto a partir dos conhecimentos tradicionais quanto da saúde pública; participar na oferta de cuidados tradicionais de saúde, bem como na provisão de serviços no subsistema de atenção à saúde indígena; atuar em ações de prevenção, promoção, tratamento e reabilitação nas diversas fases da vida; desenvolver ação política e comunitária em sua área de atuação; compreender a organização do SUS e do Subsistema de Saúde Indígena, motivando a comunidade em prol de ações em saúde; articular-se com outros setores da sociedade para resolver os problemas sanitários da comunidade; participar das atividades de controle social; planejar, executar e avaliar ações em saúde, com destaque para as práticas de educação em saúde; contribuir com o movimento indígena para encaminhar lutas pela melhoria da saúde.

O desenvolvimento de tais estratégias exige que conhecimentos científicos e nativos previamente sistematizados sejam acionados para orientar o processo formativo. Demanda igualmente o desenvolvimento de pesquisaação direcionada a temáticas necessárias à formação, que sejam capazes de suprir lacunas do conhecimento já disponível e de orientar as respostas 
cotidianas dos agentes de saúde frente às necessidades das comunidades, contribuindo para a redução de situações de vulnerabilidade.

Em janeiro de 2009, realizou-se uma etapa piloto do curso técnico profissionalizante, na comunidade de Vila Nova, no rio Xié, afluente do rio Negro. Na ocasião, foram trabalhados conteúdos dos eixos curriculares 'cultura' e 'território'. No primeiro, a ênfase se deu na análise de processos tradicionais de trabalho que garantem a reprodução da vida social e no reconhecimento de agravos e doenças a eles relacionados; também foram discutidas as características da organização social das culturas indígenas locais e de seus sistemas tradicionais de doença-cura-cuidados. No âmbito do eixo território, os educandos empreenderam um minucioso mapeamento dos recursos naturais e sociais disponíveis em suas áreas de atuação. Os produtos dessa atividade subsidiaram a análise das relações entre as condições de vida e a promoção da saúde. Tais atividades foram desenvolvidas em permanente interlocução entre os professores, agentes de saúde e velhos indígenas conhecedores da cultura que participaram do curso aportando informações mito-cosmológicas aos conteúdos nele trabalhados.

A experiência propiciou um importante momento de reflexão para educandos e docentes, permitindo avaliar a pertinência e viabilidade do uso de estratégias e conteúdos pedagógicos priorizados. As primeiras impressões foram bastante positivas, com destaque para o alto grau de envolvimento e comprometimento dos Acis com o curso, em que pese algumas dificuldades enfrentadas por eles, em função de sua baixa escolaridade.

\section{Considerações finais}

A experiência aqui relatada surge como uma das respostas possíveis às demandas das organizações e lideranças indígenas rionegrinas que, ao longo das últimas décadas, protagonizam importante luta pela elevação da escolaridade e formação profissional de seus pares nas áreas da educação e saúde indígena. Além disso, espera-se que este curso possa subsidiar políticas públicas que atendam às especificidades da educação e saúde indígenas.

A proposta enfrenta complexos desafios, seja na construção de saberes na interface entre conhecimentos científicos e tradicionais, seja no exercício da docência em contexto intercultural e multilinguístico. Nesse sentido, a participação de alunos, moradores das comunidades e velhos sábios indígenas que compartilharam a experiência pedagógica com os professores nãoindígenas mostrou-se elemento fundamental no aprimoramento da relação ensino-aprendizado. Foi uma estratégia que impactou positivamente a participação dos agentes de saúde, contribuindo para o reconhecimento dos saberes tradicionais como componente essencial do processo formador. 
A tais desafios somam-se a dificuldade logística para a realização das etapas formativas e o elevado dispêndio financeiro. As grandes dimensões geográficas da região demandam o deslocamento de professores e educandos para os pólos de formação onde as atividades didáticas se desenvolvem. A logística envolve também a organização de alojamento e alimentação para os participantes, por períodos que variam entre duas a três semanas, duas a três vezes ao ano, multiplicados pelos cinco pólos de formação, o que eleva consideravelmente os custos de cada etapa formativa.

A experiência está ainda em andamento, mas as avaliações até aqui empreendidas apontam para o fortalecimento do trabalho dos agentes de saúde, pautado, entre outros fatores, pelo reconhecimento da profissão e do trabalhador indígena; pela melhor definição das atividades e do perfil de responsabilidades e atribuições dos Acis; pela melhoria da qualidade do trabalho e da atenção à saúde das populações; e pela maior satisfação dos líderes do movimento indígena, visto que o projeto responde a uma das principais prioridades do movimento indígena, que é o acesso à escolaridade diferenciada.

Nesse contexto, o processo formador não representa a única forma de atuação político-sanitária dos agentes políticos envolvidos; porém, pode se constituir numa importante via de apoio à produção social de sujeitos capazes de intervir e aprimorar o subsistema de saúde indígena e sua interface com o SUS.

\section{Notas}

1 Professora adjunta da Universidade Federal do Amazonas (Ufam) e pesquisadora do Centro de Pesquisa Leônidas \& Maria Deane, da Fundação Oswaldo Cruz (CpqL\&MD/ Fiocruz), Manaus, Amazonas, Brasil. Doutora em Ciências Sociais pela Universidade Estadual de Campinas (Unicamp). <luiza.garnelo@amazonia.fiocruz.br>

Correspondência: Centro de Pesquisas Leônidas \& Maria Deane, Rua Teresina, 476, Adrianópolis, Manaus, Amazonas, Brasil, CEP: 69057-070.

2 Professor adjunto da Universidade do Estado do Amazonas (UEA), Manaus, Amazonas. Mestre em Sociedade e Cultura na Amazônia pela Universidade Federal do Amazonas (Ufam). <willy.rocha@hotmail.com>

3 Professor-pesquisador da Escola Politécnica de Saúde Joaquim Venâncio, da Fundação Oswaldo Cruz (EPSJV/Fiocruz), Rio de Janeiro, Brasil. Doutor em Geografia pela Universidade Federal do Rio de Janeiro (UFRJ). <ppeiter@fiocruz.br>

4 Bolsista da Fundação de Amparo à Pesquisa do Estado do Amazonas (Fapema) no Centro de Pesquisa Leônidas \& Maria Deane, da Fundação Oswaldo Cruz (CpqL\&MD/ 
Fiocruz), Manaus, Amazonas, Brasil. Bacharel em Ciências Sociais pela Universidade Federal do Amazonas (Ufam). <sullysampaio@amazonia.fiocruz.br>

5 Coordenadora Pedagógica do Curso Técnico de Agente Comunitário Indígena de Saúde (Acis) do Centro de Pesquisa Leônidas \& Maria Deane, da Fundação Oswaldo Cruz (CpqL\&MD/Fiocruz), Manaus, Amazonas, Brasil. Mestre em Educação pela Universidade Federal do Amazonas (Ufam). <elciclei@amazonia.fiocruz.br>

6 Professora-pesquisadora do Laboratório de Educação Profissional em Atenção em Saúde da Escola Politécnica de Saúde Joaquim Venâncio, da Fundação Oswaldo Cruz (EPSJV/Fiocruz), Rio de Janeiro, Brasil. Doutoranda em Saúde Pública pela Escola de Saúde Pública Sergio Arouca, da Fundação Oswaldo Cruz (Ensp/Fiocruz). <analupontes@fiocruz.br>

7 Professora-pesquisadora da Escola Politécnica de Saúde Joaquim Venâncio, da Fundação Oswaldo Cruz (EPSJV/Fiocruz), Rio de Janeiro, Brasil. Doutora em Educação pela Pontifícia Universidade Católica do Rio de Janeiro (PUC-RJ). <anakstauffer@fiocruz.br>

\section{Referências}

BRASIL. Ministério da Saúde. Funasa. Departamento de Saúde Indígena. Formação de agentes indigenas de saúde. Brasília, 1999.

Ministério da Saúde. Funasa. Distrito Sanitário Especial Indígena do Rio Negro. Relatório de Gestão. São Gabriel da Cachoeira, 2003.

Ministério da Saúde. Ministério da Educação. Referencial curricular para curso técnico de agente comunitário de saúde. Série A. Normas e Manuais Técnicos. 2. ed. rev. Brasília: Ministério da Saúde, 2005.

BUCHILlET, Dominique. Contas de vidro, enfeites de branco e 'potes de malária'. Brasília: Editora UNB, 1995.

CANÁRIO, Rui. Formação e mudança no campo da saúde. In: CANÁRIO, R. Formação e situações de trabalho. Lisboa: Porto Editora, 2003.

CASTRO, Claudia de. Fronteira das culturas: educação profissional na saúde indígena: História, impasses e perspectivas da formação do agente de saúde indígena. Revista RET-SUS, ano I, n. 8, p. 4-7, maio 2005.
CECCIM, Ricardo Burg; FERLA, Alcindo Antônio. Educação e saúde: ensino e cidadania como travessia de fronteiras. Trabalho, Educação e Saúde, v. 6, n. 3, p. 573-598, 2008-2009.

CIAVATTA, Maria. Formação integrada: a escola e o trabalho como lugares de memória e identidade. In: FRIGOTTO, Gaudêncio; CIAVATTA, Maria; RAMOS, Marise (Orgs.). Ensino Médio integrado: concepção e contradições. São Paulo: Cortez, 2005.

ESCOLA POLITÉCNICA DE SAÚDE JOAQUIM VENÂNCIO. Projeto politicopedagógico. Rio de Janeiro: Fiocruz, 2005.

GARNELO, Luiza. Cosmologia, ambiente e saúde: mitos e ritos alimentares Baniwa. História, Ciências, Saúde: Manguinhos, v. 14, p. 191-212, 2007. Suplemento.

- Poder, hierarquia e reciprocidade: saúde e harmonia entre os Baniwa do Alto Rio Negro. Coleção Saúde dos Povos Indígenas. Rio de Janeiro: Editora Fiocruz, 2003.

GARNELO, Luiza; WRIGHT, Robin. Doença, cura e serviços de saúde. Representações, 
práticas e demandas Baniwa. Cadernos de Saúde Pública, v. 17, n. 2, p. 273-284, 2001.

GONDIM, Gracia Maria de Miranda. Interdisciplinaridade e autonomia: a concepção unitária no currículo do curso técnico de Vigilância em Saúde da EPSJV/Fiocruz. In: PEREIRA, Isabel Brasil; DANTAS, André Vianna (Orgs.). Estudos de politecnia e saúde. Rio de Janeiro: Escola Politécnica de Saúde Joaquim Venâncio, 2009.

HILL, Jonathan. Social equality and ritual hierarchy: The Arawakan Wakuenai of Venezuela. American Ethnologist, v. 11, n. 3, p. 528-544, 1984.

INSTITUTO SOCIOAMBIENTAL. Ministério do Meio Ambiente. Avaliação e identificação de ações prioritárias para a conservação, utilização sustentável e repartição dos benefícios da biodiversidade na Amazônia brasileira. Brasília: MMA/SBF, 2001. 1 CD-ROM.

MONKEN, Maurício; BARCELLOS, Christovam. O território na promoção e Vigilância em Saúde: o território e o processo saúdedoença. Rio de Janeiro: EPSJV/Fiocruz, 2007.

MONKEN, Maurício. Contexto, território e o processo de territorialização de informações: desenvolvendo estratégias pedagógicas para a educação profissional em Vigilância em Saúde. In: BARCELLOS, Christovam (Org.). A geografia e o contexto dos problemas de saúde. Rio de Janeiro: Abrasco/Icict/EPSJV, 2008.
PEREIRA, Isabel Brasil; RAMOS, Marise Nogueira. Educação profissional em saúde. Rio de Janeiro: Editora Fiocruz, 2006.

RAMOS, Marise Nogueira. É possível uma pedagogia das competências contra-hegemônica? Relações entre pedagogia das competências, construtivismo e neopragmatismo. Trabalho, Educação e Saúde, v. 1, n. 1, 2003.

ROCHA, Esron. Uma etnografia das práticas sanitárias no Distrito Sanitário Especial Indígena do Rio Negro: noroeste do Amazonas. 2007. Dissertação de Mestrado em Sociedade e Cultura na Amazônia, Manaus: Instituto de Ciências Humanas e Letras, Universidade Federal do Amazonas, 2007.

SANTOS, Milton. A natureza do espaço: técnica e tempo. Razão e emoção. São Paulo: Hucitec, 1997.

TEIXEIRA, Carmem Fontes; PAIM, Jairnilson Silva; VILASBOAS, Ana Luiza. SUS, modelos assistenciais e vigilância da saúde. Iesus, v. VII, n. 2, p. 7-28, 1998.

TEIXEIRA, Carmem Fontes; SOLLA, Jorge Pereira. Modelo de atenção à saúde: promoção, vigilância e saúde da família. Salvador: EDUFBA, 2006.

WRIGHT, Robin. História indígena do noroeste da Amazônia: hipóteses, questões e perspectivas. In: CARNEIRO DA CUNHA, Manuela (Org.). História dos indios no Brasil. São Paulo: Companhia das Letras, p. 253-268, 1992.

Recebido em 05/05/2009

Aprovado em 25/05/2009 
